Nervenarzt 2022 · 93:499-502

https://doi.org/10.1007/s00115-021-01212-y

Angenommen: 7. September 2021

Online publiziert: 13. Oktober 2021

(c) Der/die Autor(en) 2021

\section{COVID-19-Impfungen im Maßregelvollzug: einige Überlegungen zu ethischen und juristischen Aspekten}

\author{
Peter Praus ${ }^{1,2} \cdot$ Eva Biebinger $^{2} \cdot$ Harald Dreßing ${ }^{1}$ \\ 'Klinik für Psychiatrie und Psychotherapie, J5, Zentralinstitut für Seelische Gesundheit, Mannheim, \\ Deutschland \\ ${ }^{2}$ Klinik für Forensische Psychiatrie, Pfalzklinikum für Neurologie und Psychiatrie, Klingenmünster, \\ Deutschland
}

\section{Hintergrund}

Ohne Frage verbinden sich mit der zunehmenden Inanspruchnahme wirksamer Impfungen gegen COVID-19 Hoffnungen auf ein absehbares Ende der Coronaviruspandemie und den damit einhergehenden Einschränkungen von Grundrechten. Gerade in Kliniken des Maßregelvollzuges haben letztere im Interesse des Infektionsschutzes zu teils erheblichen Veränderungen der Behandlungsabläufe, der Durchführung von Lockerungen und somit - vor dem Hintergrund des Verhältnismäßigkeitsgrundsatzes - sowohl juristischen als auch ethischen Dilemmata geführt $[15,21]$. Mittlerweile liegen nun, beruhend auf den Erfahrungen des bayerischen Maßregelvollzuges, belastbare, strukturierte Handlungsempfehlungen vor, die sowohl Infektionsschutz als auch Patientenrechte und Sicherheitsaspekte umfassend berücksichtigen und auf die für das Management künftiger Pandemien im Maßregelvollzug zurückgegriffen werden kann [8]. Daten aus dem Strafvollzug der Vereinigten Staaten von Amerika sowie aus Gefängnissen in England und Wales legen übereinstimmend nahe, dass die COVID-19-bedingte Mortalität in den jeweiligen Einrichtungen gegenüber der COVID-19-Mortalität in der Allgemeinbevölkerung in etwa um den Faktor 3 erhöht war $[2,19]$, was die enorme Bedeutung derartiger Maßnahmen in Einrichtungen des Straf- und Maßregelvollzuges unterstreicht. Aktuelle Daten aus dem Strafvollzug in Rhode Island, Vereinigte Staaten von Amerika, zeigen, dass mit den aktuell verfügbaren Impfstoffen bei gleichzeitig nur sehr selten beobachteten "Impfdurchbrüchen" diesem dramatischen Befund abgeholfen werden könnte [3]. Nachvollziehbar ist somit auch, dass sowohl auf ethischer Grundlage als auch im Interesse des Infektionsschutzes eine Priorisierung inhaftierter bzw. untergebrachter Personen im Zuge nationaler Impfkampagnen gegen COVID-19 geboten ist [7, 20].

\section{Situation in der Bundesrepublik Deutschland}

Während der Deutsche Ethikrat bereits im Februar 2021 Überlegungen zu der Frage veröffentlichte, wie der mögliche Wegfall der Gründe für den Entzug individueller Freiheitsrechte infolge einer wirksamen Impfung unter normativen Gesichtspunkten mit dem Bedürfnis der Allgemeinheit nach Schutz vor einer COVID-19Infektion und Gleichbehandlung in Einklang gebracht werden könnte, wurden auch explizit marginalisierte, in besonderem Maße schutzbedürftige Gruppen in die o.g. Überlegungen mit einbezogen (https://www.ethikrat.org/fileadmin/ Publikationen/Ad-hoc-Empfehlungen/ deutsch/ad-hoc-empfehlung-besondereregeln-fuer-geimpfte.pdf). Konkret heißt es hierzu:

Aufgrund der Isolationsmaßnahmen sind die in Pflege-, Senioren-, Behinderten- und Hospizeinrichtungen Lebenden noch immer Belastungen ausgesetzt, die erheblich über das 
hinausgehen, was andere Bürgerinnen und Bürger erdulden müssen. Diese Sonderbelastung ist nur zu rechtfertigen, solange die in solchen Einrichtungen Lebenden noch nicht geimpft sind. Sie gehören unter anderem deshalb zur ersten Gruppe, die derzeit geimpft wird.

Bemerkenswerterweise blieben hier die spezifischen Gegebenheiten hinsichtlich der Bedürfnisse und Freiheitsrechte von im Maßregelvollzug untergebrachten Patienten - immerhin ein relevanter Teil aller in psychiatrischen Kliniken behandelter Patienten [11] - letztlich unberücksichtigt, obwohl sie ähnlichen Lebensbedingungen und Beschränkungen der Freiheits- und Besuchsrechte wie in Pflegeeinrichtungen untergebrachte Personen ausgesetzt waren und ebenfalls ein erhöhtes Sterberisiko aufweisen. Auch psychisch schwer erkrankte Patienten, die in allgemeinpsychiatrischen Versorgungsstrukturen behandelt werden, wurden im Rahmen der nationalen deutschen Impfstrategie zunächst nicht mit einer prioritären Impfempfehlung bedacht [4], obwohl sie einem nachweislich erhöhten Risiko ausgesetzt sind, infolge einer COVID-19-Erkrankung zu versterben [17]. Später wurde dann eine Priorisierung vergleichsweise schwerer erkrankter allgemeinpsychiatrischer Patienten empfohlen; schutzbedürftige Patienten im Maßregelvollzug erhielten jedoch bei anhaltender Knappheit der verfügbaren Impfstoffe weiterhin keine explizite Empfehlung zum bevorzugten Angebot einer COVID-19-Impfung. Die sich hieraus ergebende faktische Diskrepanz in der Behandlung psychisch erkrankter Menschen mutet angesichts der o.g. Befunde jedoch artifiziell an.

Im internationalen Vergleich ist erwähnenswert, dass Ende Dezember 2020 auch $47 \%$ der Bundesstaaten der Vereinigten Staaten von Amerika keine spezifische Empfehlung dazu abgaben, wann unter Gefängnisbedingungen lebende Personen im Verlauf der Phasen der nationalen Impfkampagne ein Impfangebot erhalten sollten [22], obwohl deren erhöhtes Risiko infolge einer COVID-19-Erkrankung zu versterben bereits bekannt war [19].

Mit der Möglichkeit einer wirksamen Impfung gegen COVID-19 für im Maßregelvollzug untergebrachte Patienten verbin- det sich aus der Sicht der Autoren nun einerseits die Hoffnung, dass Problemfelder, die einen direkten Bezug zur Pandemiesituation aufweisen, und eine Knappheit an Impfstoffen an Relevanz verlieren werden. Andererseits ergeben sich hierdurch möglicherweise auch neue Fragestellungen von praktischer Relevanz, die mittelfristig an Bedeutung gewinnen könnten.

\section{Einige relevante Problemfelder}

\section{Einwilligungsfähigkeit}

Während wohl kaum eine Debatte darüber entstehen dürfte, ob gemäß $\S 64$ StGB untergebrachte Patienten mehrheitlich einwilligungsfähig hinsichtlich der Applikation einer Impfung gegen COVID-19 sein könnten, so stellt sich die Situation in der Maßregel gemäß § 63 StGB grundlegend anders dar, wo ein wesentlicher Teil der Untergebrachten an einer Schizophrenie erkrankt ist [10]. Insbesondere mit der Beurteilung der Einwilligungsfähigkeit in medizinische Maßnahmen sind jedoch hohe Anforderungen verbunden [5], denen Patienten mit chronischen, willensbestimmenden Krankheitssymptomen mitunter nicht gerecht werden. Doch weisen gerade an einer Schizophrenie erkrankte Patienten eine erhöhte COVID-19-Mortalität auf [13]. Gerade hier sind also eine engmaschige Zusammenarbeit mit gesetzlichen Betreuern und eine gründliche psychiatrische Befunderhebung im Hinblick auf die Einwilligungsfähigkeit der Patienten unbedingt geboten. Impfungen gegen den natürlichen Willen dieser überdurchschnittlich häufig Zwangsmaßnahmen unterworfenen Patientengruppe [18] erscheinen aus der Sicht der Autoren derzeit unter ethisch-moralischen Gesichtspunkten kaum begründbar. Andererseits kommt gerade Behandlern in der forensischen Psychiatrie eine wesentliche Rolle bei der Akzeptanz von Impfungen bei forensisch untergebrachten Patienten zu [1]. Dies setzt allerdings auch voraus, dass COVID-19-Impfungen unter Mitarbeitern der forensischen Psychiatrie eine hohe Akzeptanz aufweisen. Ferner wird die Frage zu beantworten sein, wie unter den Bedingungen des Maßregelvollzuges angesichts der bekannten Infektionsrisiken mit Patienten verfahren werden soll, die aufgrund einer freien Willensbildung eine COVID-19Impfung ablehnen. Zu diesem Thema liegen mittlerweile erste Diskussionsbeiträge vor [12].

\section{Besondere Patientengruppen}

Aktuelle Daten aus dem Vereinigten Königreich [9] weisen darauf hin, dass forensisch untergebrachte Patienten mit einem Migrationshintergrund, die auch in Deutschland im Vergleich zu ihrem Anteil an der Gesamtbevölkerung überdurchschnittlich häufig in forensisch-psychiatrischen Kliniken untergebracht sind [16], wahrscheinlich häufiger eine Impfung gegen COVID19 ablehnen als andere Patienten. Hier wird in der Beratung und Aufklärung besonders auf kultursensible Aspekte der forensischen Therapie und etwaige Sprachbarrieren zu achten sein. Möglicherweise spielen auch Sozialisationsbedingungen in wirtschaftlich benachteiligten Regionen, Bürgerkriegsgebieten usw., wo Gesundheitsvorsorge und Impfungen gegebenenfalls eine geringere Bedeutung beigemessen wird, bei der Ablehnung einer COVID-19Impfung eine Rolle. Es wird also darauf ankommen, eine im Vergleich schlechtere Beratung und Versorgung dieser Patientengruppe unbedingt zu vermeiden. In aktuellen Konsensuspapieren wird eine Verbesserung der Versorgung gerade dieser Patienten ausdrücklich angemahnt [14]. Möglicherweise könnte hier sprachunabhängiges Informationsmaterial dazu beitragen, Barrieren beim Zugang zu effektiven medizinischen Maßnahmen wie Impfungen zu reduzieren [6].

\section{Rehabilitationsperspektiven}

Grundsätzlich erscheint auch die Frage berechtigt, inwiefern die Ablehnung einer COVID-19-Impfung oder eine diesbezüglich mangelnde Einwilligungsfähigkeit Auswirkungen auf die gesellschaftliche Rehabilitation außerhalb und die Behandlung dieser Patienten innerhalb forensischer Kliniken, beispielsweise in Bezug auf die Durchführung von Gruppentherapien, Lockerungen, Erprobungen und weitere vollzugsöffnende Maßnahmen, haben könnte. Derzeit werden für nicht gegen COVID-10 geimpfte Personen Beschränkungen des Zugangs zu bestimmten 
Aspekten des öffentlichen Lebens diskutiert und digitale Gesundheitsnachweise eingeführt. Hier wird einer möglichen Benachteiligung dieser Patienten oder einer Verzögerung des Behandlungsverlaufes im Zusammenhang mit Maßnahmen des Infektionsschutzes im Vergleich mit anderen Patienten, die eine Impfung erhalten haben, vorzubeugen sein. Darüber hinaus sind Auswirkungen auf die Möglichkeiten, Plätze in komplementären Wohneinrichtung mit dem Ziel der Durchführung einer Dauererprobung zu organisieren oder die Integration in einen Werkstattbetrieb zu ermöglichen, abzuwarten.

\section{Schlussbemerkung}

Die oben aufgeführten Fragestellungen sind mitnichten als erschöpfend dargestellt zu betrachten, sondern sollen aus der Sicht der Autoren lediglich einen ersten Beitrag zur Diskussion spezifischer Fragestellungen im Hinblick auf COVID-19Impfungen im Maßregelvollzug darstellen. Darüber hinaus sind viele weitere Fragen von direkter praktischer Relevanz denkbar: Wie sollte man beispielsweise damit umgehen, wenn Patienten bestimmte Impfstoffe ablehnen oder in einer spezifischen Kliniknur Impfstoffe zur Verfügung gestellt werden, die von der ständigen Impfkommission des Paul-Ehrlich-Instituts nicht für alle Altersgruppen empfohlen werden? Zusammenfassend zeigen sich hier also einmal mehr die großen Herausforderungen, denen Patienten und Behandler im Maßregelvollzug im Zusammenhang mit der COVID-19-Pandemie ausgesetzt sind.

Bisherige Erfahrungen an der Klinik für Forensische Psychiatrie des Pfalzklinikums weisen auf eine gute Annahme des Impfangebotes unter Patienten und Mitarbeitern hin (Stand Ende August 2021). Aufklärungs- und Beratungsgespräche mit an einer Schizophrenie erkrankten Patienten gestalteten sich erwartungsgemäß aufwendiger. Generelle, patientenunabhängige Schwierigkeiten hinsichtlich Beratung und Aufklärung ergaben sich daraus, dass noch Anfang Juni 2021 seitens der zuständigen Behörden keine Zusagen hinsichtlich des konkret zum Einsatz kommenden Impfstoffes erhältlich waren, was auch auf die eingangs erwähnte Knappheit der Impfstoffe und das Fehlen einer konkreten Priorisierungsempfehlung zurückzuführen gewesen sein mag.

Angesichts der eingangs skizzierten Befundlage wird im Zuge künftiger Pandemien also besonders auf die Bedürfnisse und Rechte marginalisierter Personengruppen, die unter den Bedingungen einer gerichtlichen Unterbringung leben, zu achten sein.

\section{Fazit für die Praxis}

- Es liegen mittlerweile praktisch bewährte Empfehlungen zum Pandemiemanagement in der forensischen Psychiatrie vor.

- Bei der Beratung der Patienten sind deren Einwilligungsfähigkeit und Schutzbedürftigkeit unbedingt zu beachten.

- Behandler können die Entscheidung ihrer Patienten für eine Impfung dabei positiv beeinflussen.

- Patienten mit Migrationshintergrund sind im Hinblick auf den Zugang zu Impfungen möglicherweise benachteiligt.

- Rehabilitationsperspektiven von Patienten, die keine Impfung erhalten haben, müssen wo immer möglich gewahrt bleiben.

- Einer Benachteiligung forensischer Patienten muss im Zuge künftiger Pandemien bewusst entgegengewirkt werden.

Korrespondenzadresse

Dr. med. Peter Praus

Klinik für Psychiatrie und Psychotherapie, J5, Zentralinstitut für Seelische Gesundheit 68159 Mannheim, Deutschland peter.praus@zi-mannheim.de

Funding. Open Access funding enabled and organized by Projekt DEAL.

Interessenkonflikt. P. Praus, E. Biebinger und H. Dreßing geben an, dass kein Interessenkonflikt besteht.

Open Access. Dieser Artikel wird unter der Creative Commons Namensnennung 4.0 International Lizenz veröffentlicht, welche die Nutzung, Vervielfältigung, Bearbeitung, Verbreitung und Wiedergabe in jeglichem Medium und Format erlaubt, sofern Sie den/die ursprünglichen Autor(en) und die Quelle ordnungsgemäßnennen, einen Link zur Creative Commons Lizenz beifügen und angeben, ob Änderungen vorgenommen wurden.

Die in diesem Artikel enthaltenen Bilder und sonstiges Drittmaterial unterliegen ebenfalls der genannten Creative Commons Lizenz, sofern sich aus der Abbildungslegende nichts anderes ergibt. Sofern das betreffende Material nicht unter der genannten Creative Commons Lizenz steht und die betreffende Handlung nicht nach gesetzlichen Vorschriften erlaubt ist, ist für die oben aufgeführten Weiterverwendungen des Materials die Einwilligung des jeweiligen Rechteinhabers einzuholen.
Weitere Details zur Lizenz entnehmen Sie bitte der Lizenzinformation auf http://creativecommons.org/ licenses/by/4.0/deed.de.

\section{Literatur}

1. Borthwick C, O'connor R, Kennedy L (2021) Predicting and understanding seasonal influenza vaccination behaviour among forensic mental health inpatients. Psychol Health 36(10):1235-1259. https://doi.org/10.1080/08870446.2020.1846038

2. Braithwaite I, Edge C, Lewer D et al (2021) High COVID-19 death rates in prisons in England and Wales, and the need for early vaccination. Lancet Respir Med 9:569-570

3. Brinkley-Rubinstein L, Peterson M, Martin R et al (2021) Breakthrough SARS-CoV-2 infections in prison after vaccination. $\mathrm{N}$ Engl $\mathrm{J}$ Med 385(11):1051-1052. https://doi.org/10.1056/ NEJMc2108479

4. De Picker LJ, Dias MC, Benros ME et al (2021) Severe mental illness and European COVID-19 vaccination strategies. Lancet Psychiatry 8:356-359

5. Dreßing H (2020) Zivilrechtliche Grundlagen in der Psychiatrie. Psych Up2date 14:523-536

6. Dutilleul A, Morel J, Schilte C et al (2019) How to improve vaccine acceptability (evaluation, pharmacovigilance, communication, public health, mandatory vaccination, fears and beliefs). Therapie 74:131-140

7. Fiore V, De Vito A, Madeddu G et al (2021) SARSCoV-2 vaccination among incarcerated people: a barrier to overcome. Front Public Health 9:704520

8. Gaudernack D, Dudeck M (2020) COVID-19 in forensic psychiatric hospitals in bavaria: finding a balance between infection prevention, collective security and patient rights. Forensic Sci Int Mind Law 1:100035

9. Gibbon S, Mcphail E, Mills G et al (2021) Uptake of COVID-19 vaccination in a medium secure psychiatric hospital population. BJPsych open 7:e108

10. Habermeyer E, Wolff R, Gillner M et al (2010) Patienten mit schizophrenen Störungen im psychiatrischen Maßregelvollzug. Nervenarzt 81:1117-1124

11. Leygraf $N$ (2018) Behandlung im Maßregelvollzug. Forens Psychiatr Psychol Kriminol 12:91-92

12. Liebrenz M, Bhugra D, Buadze A et al (2021) Prisoner's dilemma: ethical questions and mental health concerns about the COVID-19 vaccination and people living in detention. Forensic Sci Int Mind Law 2:100044

13. Mohan M, Perry BI, Saravanan P et al (2021) COVID-19 in people with schizophrenia: potential mechanisms linking schizophrenia to poor prognosis. Front Psychiatry 12:684

14. Müller JL, Saimeh N, Briken P et al (2017) Standards für die Behandlung im Maßregelvollzug nach §§ 63 und 64 StGB. Nervenarzt 88:1-29

15. Praus $P$, Biebinger $E$, Dressing $H$ (2021) COVID19: challenges for forensic therapy and quality of treatment in institutions for forensic commitment. Nervenarzt. https://doi.org/10.1007/s00115-02101102-3

16. Querengässer J, Traub H-J (2019) Nichtdeutsche Staatsbürger im Maßregelvollzug gemäß § 64 StGB-Jahres- und Bundesländervergleich der Neuanordnungen 2010-2015 sowie Gruppenbesonderheiten. Forens Psychiatr Psychol Kriminol 13:251-260 
17. Reilev M, Kristensen KB, Pottegard A et al (2020) Characteristics and predictors of hospitalization and death in the first 11122 cases with a positive RTPCR test for SARS-CoV-2 in Denmark: a nationwide cohort. Int JEpidemiol 49:1468-1481

18. Reinwald JR, Horten B, Dreßing $\mathrm{H}$ et al (2021) Strukturmerkmale und Anwendungshäufigkeit von Zwangsmaßnahmen im deutschen Maßregelvollzug. Psychiatr Prax. https://doi.org/10.1055/a1391-0192

19. Saloner B, Parish K, Ward JA et al (2020) COVID-19 cases and deaths in federal and state prisons. JAMA 324:602-603

20. Simpson PL, Levy M, Butler T (2021) Incarcerated people should be prioritised for covid-19 vaccination. BMJ373:n859

21. Steinböck H (2020) Maßregelvollzug in den Zeiten derCorona-pandemie. RechtPsychiatr 38:131-134

22. Strodel R, Dayton L, Garrison-Desany HM et al (2021) COVID-19 vaccine prioritization of incarcerated people relative to other vulnerable groups: An analysis of state plans. PLoS ONE 16:e253208

\section{Gerhard Gründer \\ Psychopharmaka absetzen? Warum, wann und wie?}

\section{Urban \& Fischer/Elsevier 2022, 113 S., (ISBN: 978-3-437-23585-6), 29 EUR}

Auf schlanken 113 Seiten lässt uns Prof. Gründer an seinem in Breite und Tiefe beeindruckenden Wissen über psychopharmakologische Grundlagen und klinische Studien teilhaben. Fallbeispiele und viele praktische Hinweise runden das Buch ab. Der Bogen spannt sich von den Antidepressiva über die Neuroleptika, die Benzodiazepine bis zu Lithium.

Wer Prof. Gründer kennt, weiß, dass er bis vor einigen Jahren seine Expertise häufig dazu nutzte, kritische Stellungnahmen zu Psychopharmaka unmittelbar zurückzuweisen und hierbei gelegentlich in den Verdacht geriet, zu sehr das Lied der pharmazeutischen Industrie zu singen. Seine vielfältigen finanziellen Beziehungen zur Industrie bestehen bis heute. Noch 2013 hatte Gründer eine DGPPNPresseinformation der mit dem unzutreffenden und in seiner Wortwahl irritierenden Titel „Antidepressiva helfen Selbstmorde zu verhindern" gerechtfertigt und negative Berichterstattung über Psychopharmaka abgelehnt, da sie Menschen verunsicheren und ihnen schaden würde.

Umso erstaunlicher ist die kritische Haltung, die Gründer seit wenigen Jahren zeigt, und noch mehr die schonungslose Zusammenstellung höchst beunruhigender Befunde zu Langzeiteffekten von Psychopharmaka. Es verwundert nicht, dass Gründer im Einleitungskapitel Kollegen mit den Worten zitiert, er hätte sich „vom Saulus zum Paulus" gewandelt. Nunmehr beginnt Gründer, dass es der Glaubwürdigkeit und der Reputation des Faches Psychiatrie schade, nicht offen über langfristige negative Folgen einer Psychopharmakotherapie zu sprechen. Fast in Form einer Generalabrechnung legt er dann dar, dass Antidepressiva und Neuroleptika nicht nur Absetz- und Entzugssymptome hervorrufen, sondern aufgrund von Toleranzentwicklung auch Chronizität und Therapieresistenz bewirken können Folgen, die wohl auch nach dem Ende der Pharmakotherapie fortbestehen. Noch anderthalb Jahre nach dem Absetzen von Fluoxetin war in einer Affenstudie eine Erhöhung der Serotonintransporterexpression festzustellen; und die
Dopamin-Supersensitivität nach einer Neuroleptikabehandlung persistiert ebenfalls, mit einem entsprechend erhöhten Rezidivrisiko ein Befund, der eigentlich schon seit Jahrzehnten aus dem dauerhaften Fortbestehen tardiver Dyskinesien auch nach Beendigung der Neurolepsie abgeleitet werden kann. Schizophrene Negativsymptomatik und kognitive Defizite sind vermutlich häufig Artefakte einer neuroleptischen Medikation oder zumindest einer zu hohen Dosis.

Fazit: Das Buch lässt nachvollziehen, wie Gründers aufgrund seiner allumfassenden Kenntnis der Befunde zur Änderung seiner Beurteilung von Psychopharmaka kam. Seiner Forderung, schon beim Beginn einer Medikation müsse deren Beendigung mitgedacht werden, ist zuzustimmen. Er legt dar, dass die S3-Leitlinien (Depression; Schizophrenie) hier weiter sind, als die Behandlungsrealität, wenn sie vom Einsatz von Antidepressiva bei leichten Depressionen abraten und darauf hinweisen, dass eine Neuroleptika-Dosiserhöhung rückgängig zu machen ist, wenn sie keinen klinischen Mehrwert erbrachte, sowie, dass Medikamente ohne klaren Nutzen abgesetzt und nicht durch ein weiteres Pharmakon ergänzt gehören. Das kluge Buch hinterlässt erhebliche Verunsicherung und der Titel würde treffender lauten „Psychopharmaka ansetzen?".

Prof. Dr. med. Tom Bschor, Berlin 